\title{
Investigation of Sensor Data Fusions using Continuous-Time Decentralized Kalman Filters (DKF)
}

\author{
${ }^{1}$ Tushar, ${ }^{2}$ Dr. Abhijit Nayak \\ ${ }^{1} \mathrm{PhD}$ Scholar (Full Time), USICT, GGSIPU, Dwarka, New Delhi \\ ${ }^{2}$ Professor and Dean, Bhagwan Parshuram Institute of Technology, Rohini, New Delhi \\ Email: tusharvardhan@yahoo.in,nayakabhijit1972@gmail.com
}

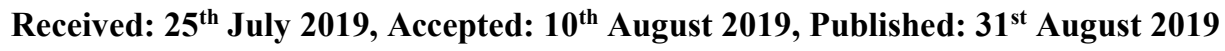

\begin{abstract}
The integration of sensors deals with the accumulation of information from two or more sensors in network. However the accumulation of data is typically the subject of research in the area of image analysis. Generally the reliability of collected data might induce the problem of reconstructing to get its original form. The Various fusion technique has already been implement for text data, image data and video data. The general exploration in the sensor data fusion is to understand the behavioral pattern reformation of real time capturing with sense of accuracy. This also handle out the problems of localizing, navigation and tracing problem. This paper is to study of Kalman Filters technique with their key feature of State Vector Fusion, Measurement Fusion, Gain Fusion. The mathematical formulation for the data fusion of sensors derived through the State Vector Fusion (SVF) and covariance time propagations. The present investigation is to analysis of process of dynamically maintaining a model of the local external environment and deep exploration of fusion technique using time decentralized Kalman Filters (DKF). Fusion of perceptual information is at the heart of data fusion process.
\end{abstract}

\section{Keywords}

Sensor Fusion, Localizing, Navigation and Tracing Problem, Data Accumulation, Simultaneous Localization and Mapping (SLAM), Kalman Filters

\section{Introduction}

Fusion of sensor data is expressed as the method of accumulating the gathered data from distributed sources of nodes (DS of Sensors ) to deliver the highly accurate and complete unified data about an process, Process activities, or process terminology. SDT is a combination of many traditional disciplines sensors and sensor head (Sink Node). Figure 1 shows an overview of data integration from multiple sensors. The estimated value from a single sensor point which modeled to make original view cannot be sufficient. It need to have various data accumulation from various distributed sensors. It might possible that the deployed nodes not covers the proper area, one cover the whole while other at less area as they assigned. Fusion technique is one of the reliable, accurate and real time based technique for sensor Fusion. This technique has low error in their fusion recovery data at accumulation time. In this illustration four stages has been shown below. Sensor node connected with filter and accumulate the gathered data at one place represent as data association block and finally come into fusion block with suitable algorithms used as per data better reconstruction form. Systematic analysis of gather data are presented and model obtained are descriptively reported.

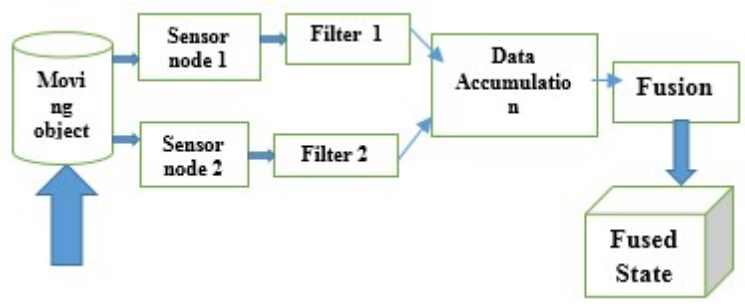

Figure 1: Concept of Multi Sensor Data Fusion

The integration of multiple sensor data turnout to be an intensive research filed in recent scientific exploration of data science [1]. Multilevel sensor send signal of captured data which gathered at central station. The all process could be analyzed as a fusion of data. If image has taken as consideration for capturing and reimaging process the quality of integration of data for image and reimaging of the data must have the significant in quality terms. The images captured from different sensor collect at central gathering station only via the signal. It distinguish because of focus on 
integrating images at fusion level. The fusion at the function level first uses the extraction function of the source data, so that the functions of each source can be used together for some purposes. The general type of fusion at the function level involves the merging of edge maps. The common type of problem occurs during integration is fusion at the signal level [3-5].

In recent time many advanced sensors reconstruct high definition images through fusion technique of gather data. So, here the focus of this paper on the integration of images gather through distributed sensor nodes. In recent years, the integration of images has attracted great attention in a wide range of applications, such as the investigation of hidden weapons [6-8], Suspected object detection [9-11], Artificial intelligent robots [12,13], medical imaginary [14,15] $[16,17]$, and military surveillance [18-20].

Models, Architectures and Frameworks

The organized structure of data gathered at central hub for integration. The theoretical framework has to contracted for the reframing of data as it could regain as original scenario. As a possible outcome, various possible pattern of may be contracted. Consequently, this must describe that how data integration algorithms can be embedded in sensor nodes with their collector base station. Currently, three main organizational models are used to describe data consolidation systems.

These are:

- Sensors and Systems Deployments

- Topological Structure

- Algorithm for Data Integration

This three interrelation of matter has the key investigation under the data fusion technique.

- Sensors and Systems Deployments: This model study the deployment strategies of sensors. The set of specific protocols for the data integration must apply over the data. It also should be the fully operational for sensors with the sink node before the final deployment. The functionality of the component owned by the system but does not make any statement regarding the implementation of the program or the creation of actual instances. The running execution and data gathering at hub operation models after the deployment is the core objective of every deployment process.

- Topological Structure: This defines topological structure as the sensor positioning coordinated of the system. This specifically pattern to the way data or information is communicated is understand under the topological configuration. The topology includes component design, connectivity and data flows. The topological structure describe the integration systems that are classified as centralized, hierarchical or distributed according to their need of requirement. It can also be specific configuration based on the systems requirement's [21-22].

- Algorithm for Data Integration: This framework consist of a set of axioms and logical interpretation of connectivity's system. Because specific a framework need with various real time application with data-rich information source to produce abstract concepts that are framed to consolidate the currently used gathered data sets. They are probabilistic configuration having proactive reason. The frame work apply over the data need very suitable approach of mathematical formulation and algorithm for finding the result and its analysis.

\section{Sensor Data Fusion (Kalman Filter) (KF)}

\section{A. State Vector Fusion}

Multi-sensor data fusion involves integration and Extract information from two or more acquired data Sensor. In various processes the data from multiple sensors must accumulate to be highly accurate with complete set of Information. In a variety of technological operation multiple sensor data fused through Kalman filtering. This is one of the best fusion technique that give better result. [23] The important one because it turned out to be a valid recursion algorithms for real-time applications. The state vectors of Kalman filter for processing data gathered at central point ( Sink node) are demonstrated for each set of observations as shown below. [26].

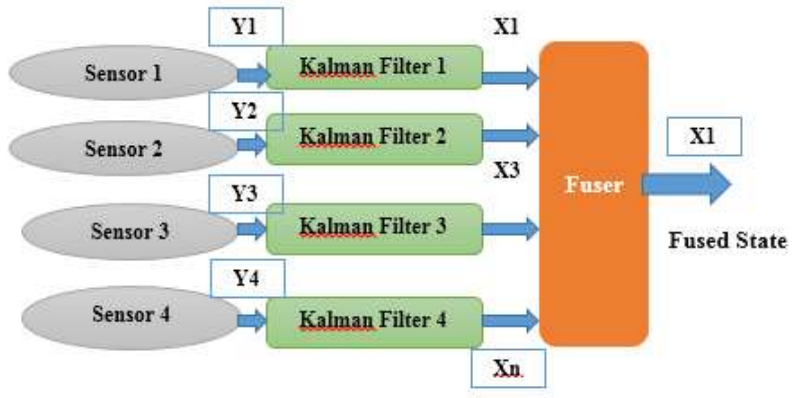

Figure 2: State Vector Fusion 
The inter-relationship of each function as below shows that state vector fusion.

$F S_{(i+1)}=\phi F S_{(i)}+G w_{(i)}$

$Z_{(i+1)}=H X_{(i)}+v_{k}$

Where

FS : Derived State Vector,

$\Psi$ : Sate Transition Matrix; $\hat{X}$

A : Gain of Matrix Data,

$w$ : Noise Estimation process,

$\mathrm{Z}$ : Estimated Vector,

$\mathrm{H}$ : Observational Matrix,

$v$ : Noise Measurement,

The Recursive algorithm developed by Kalman

The recursive algorithm derived through Kalman Filter is estimated by the equation present below. The state transition matrix equation and covariance links are described below.

$\widehat{F S}_{(i+1)}=\Psi \widehat{F S}_{(i)}$

$\widehat{P}_{(i+1)}=\Psi \hat{P}_{(i)} \Psi^{T}+G Q G^{T}$

State and covariance measurement updates are given by

$K_{(i+1)}=\breve{P}_{(i+1)\left[H \check{P}_{(i+1)} H^{T}\right]}$

$\widehat{F S}_{(i+1)}=\widetilde{F S}_{(i+1)}+K_{(i+1)}\left[Z_{(i+1)}-H \widetilde{F S}_{(i+1)}\right]$

$\hat{P}_{(i+1)}=\left[I-K_{(i+1)} H\right] \tilde{P}_{(i+1)}$

Where

$\widehat{F S}_{(i)}$ : state vector of sensor (i)

$\widehat{P}_{(i)}$ is the covariance of the sensor which is the square of difference among preferred state vector and the sensors state vector.

$A_{(i+1)}:$ Kalman Gain.

$\widehat{F S}_{(i+1)}:$ Filtered State Estimate.

$P_{(i+1)}$ : Filtered Covariance Estimate.

\section{B. Estimation of Fusion}

Estimated Function derived from the fusion method the sensor observations directly via observation model and use the proposed kalman filter to calculate the derived fused state vectors as shown below the block diagram. [23-24].

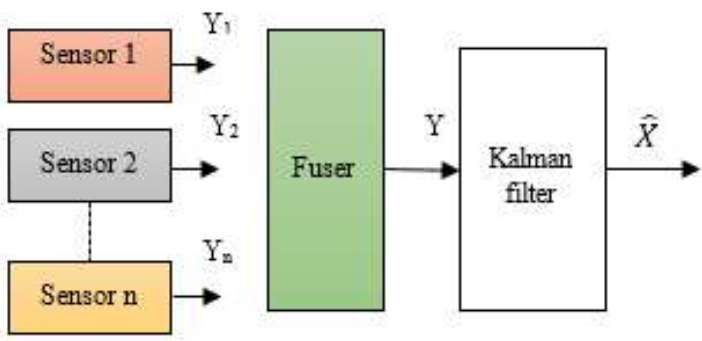

Figure 3: Measurement Fusion

The below equation demonstrate the recursive algorithm and present the covariance time propagation (Equation 8 12).

$\tilde{X}^{f_{(i+1)}}=\phi \hat{X}^{f_{i+1}}$

$\tilde{P}^{F_{i+1}}=\phi \hat{p}^{f_{(i)}} \phi^{T}+G Q G^{T}$

The state and covariance measurement data updates are given by

$K^{f_{(i+1)}}=\tilde{p}^{f_{i+1}} H^{T}\left[H P^{f_{i+1}} H^{T}+R\right]^{-1}$

$\left.\widehat{F S}^{f(i+1)}=\widehat{F S}^{f_{(i+1)}}+K^{f_{(i+1)}}\left[Z_{(i+1)}-H \tilde{X}^{f}{ }_{\left(\tilde{X}^{f}\right.}{ }_{i+1}\right)\right]$

$\hat{P}^{f(i+1)}=\left[I-K^{f}{ }_{(i+1)^{H}}\right]$

Where

$K^{f_{(i+1)}}:$ Filtered State (Fused)

$\hat{P}^{f_{(i+1)}}:$ Filtered Covariance (Fused) 


\section{Fusion Derived Gain}

The kalman recursive algorithm for gain fusion expression in equation below.

Kalman Gain $(K G)=$ Uncertainty in predicted state (UPS) / (Uncertainty in predicted state (UPS) + Uncertainty in measurement readings (UMR)).

In the process of detection of data the gain fusion algorithm derived from kalman detection in local system and established the generalized equation as below.

$\widetilde{F S}^{f(i+1)}=\Psi \tilde{X}^{f(i)}$

$\widetilde{F S}^{f(i+1)}=\Psi \widetilde{P}^{f(i)} \Psi^{T}+G Q G^{T}$

$\widetilde{F S}^{m}(i+1)=\widetilde{F S}^{f(i+1)}$

$\tilde{P}^{m_{(i+1)}}=\tilde{P}^{f_{(i+1)}}$

Where $m$ sensors counts

$\widetilde{\mathrm{FS}}^{\mathrm{f}_{(\mathrm{i}+1)}}$ : Filter state (In Fused)

$\widetilde{\mathrm{P}}^{(\mathrm{i}+1)}$ : Filter state (Fused)

\section{Conclusion}

The sensor data fusion algorithm introduced in this paper allows to combine the collected data of different sensors over time. Kalman filtering is considered at the same time as data compilation machines in local systems. This structure provides the flexibility to reconfigure the control system. New subsystems can be easily added without having to redesign the entire system. The system does not need a central processor, so if some local subsystems (each with local processor, sensors, and triggers) fail, the public system will continue to work.

\section{References}

1. Varshney, P. K. (1997). Multisensor data fusion. Electronics \& Communication Engineering Journal, 9(6), 245-253.

2. Zhang, Z., \& Blum, R. S. (1999). A categorization of multi-scale decomposition-based image fusion schemes with a performance study for a digital camera application. Proceedings of the IEEE, 87(8), 1315-1326.

3. Viswanathan, R., \& Varshney, P. K. (1997). Distributed detection with multiple sensors: Part Ifundamentals. Proceedings of the IEEE, 85(1), 54-63.

4. Blum, R. S., Kassam, S. A., \& Poor, H. V. (1997). Distributed detection with multiple sensors II. Advanced topics. Proceedings of the IEEE, 85(1), 64-79.

5. Varshney, P. K., Chen, H. M., Ramac, L. C., Uner, M., Ferris, D., \& Alford, M. (1999, October). Registration and fusion of infrared and millimeter wave images for concealed weapon detection. In Proceedings 1999 International Conference on Image Processing (Cat. 99CH36348) (Vol. 3, pp. 532-536). IEEE.

6. Uner, M. K., Ramac, L. C., Varshney, P. K., \& Alford, M. G. (1997, February). Concealed weapon detection: an image fusion approach. In Investigative Image Processing (Vol. 2942, pp. 123-132). International Society for Optics and Photonics.

7. Zhang, Z., \& Blum, R. S. (1997, March). Region-based image fusion scheme for concealed weapon detection. In Proceedings of the 31st annual conference on information sciences and systems (pp. 168-173).

8. Tu, T., Su, S. C., Shyu, H. C., \& Huang, P. S. (2001). Efficient intensity-hue-saturation-based image fusion with saturation compensation. Optical Engineering, 40.

9. Pohi, C., \& Van Genderen, J. L. (1998). Multi-sensor image fusion in remote sensing: concepts, methods and applications. I Remote Sensing, 19(5), 823-854.

10. Simone, G., Farina, A., Morabito, F. C., Serpico, S. B., \& Bruzzone, L. (2002). Image fusion techniques for remote sensing applications. Information fusion, 3(1), 3-15.

11. Abidi, M. A., \& Gonzalez, R. C. (1992). Fusion of multi-dimensional data using regularization. Data fusion in robotics and machine intelligence, 415-455.

12. Castellanos, J. A., Neira, J., \& Tardós, J. D. (2001). Multi-sensor fusion for simultaneous localization and map building. IEEE Transactions on Robotics and Automation, 17(6), 908-914.

13. Qu, G., Zhang, D., \& Yan, P. (2001, September). Medical image fusion using two-dimensional discrete wavelet transform. In Data Mining and Applications (Vol. 4556, pp. 86-95). International Society for Optics and Photonics.

14. Constantinos, S. P., Pattichis, M. S., \& Micheli-Tzanakou, E. (2001, November). Medical imaging fusion applications: An overview. In Conference Record of Thirty-Fifth Asilomar Conference on Signals, Systems and Computers (Cat. No. 01CH37256) (Vol. 2, pp. 1263-1267). IEEE. 
15. Reed, J. M., \& Hutchinson, S. (1996). Image fusion and subpixel parameter estimation for automated optical inspection of electronic components. IEEE Transactions on Industrial Electronics, 43(3), 346-354.

16. Simone, G., \& Morabito, F. C. (2001). NDT image fusion using eddy current and ultrasonic data. COMPELThe international journal for computation and mathematics in electrical and electronic engineering, 20(3), 857-868.

17. Dobeck, G. J. (1999, August). Fusing sonar images for mine detection and classification. In Detection and Remediation Technologies for Mines and Minelike Targets IV (Vol. 3710, pp. 602-614). International Society for Optics and Photonics.

18. Krebs, W. K., Scribner, D. A., Miller, G. M., Ogawa, J. S., \& Schuler, J. M. (1998, March). Beyond third generation: a sensor-fusion targeting FLIR pod for the F/A-18. In Sensor Fusion: Architectures, Algorithms, and Applications II (Vol. 3376, pp. 129-140). International Society for Optics and Photonics.

19. Murphy, R. R. (1998). Sensor and information fusion improved vision-based vehicle guidance. IEEE Intelligent Systems and their Applications, 13(6), 49-56.

20. Shahbazian, E., Bosse, E., \& Valin, P. (1998, March). Multi-agent data fusion workstation (MADFW) architecture. In Sensor Fusion: Architectures, Algorithms, and Applications II(Vol. 3376, pp. 60-68). International Society for Optics and Photonics.

21. Balci, M., \& Kuru, S. (1999, March). CORBA-based infrastructure (CORBIS) architecture for sensor data fusion systems. In Sensor Fusion: Architectures, Algorithms, and Applications III (Vol. 3719, pp. 220-229). International Society for Optics and Photonics.

22. Gan, Q., \& Harris, C. J. (2001). Comparison of two measurement fusion methods for Kalman-filter-based multisensor data fusion. IEEE Transactions on Aerospace and Electronic systems, 37(1), 273-279.

23. Yukun, C., Xicai, S., \& Zhigang, L. (2007). Research on Kalman-filter based multi-sensor data fusion. Journal of Systems engineering and Electronics, 18(3), 497-502.

24. Paik, B. S., \& Oh, J. H. (2000). Gain fusion algorithm for decentralised parallel Kalman filters. IEE Proceedings-Control Theory and Applications, 147(1), 97-103.

25. Nazar, M. S. M. (2009). A comparative study of different Kalman Filtering methods in multi sensor data fusion. In Proceedings of the International Multi-Conference of Engineers and Computer Scientists (Vol. 1, pp. 18-20).

26. Raol, J. R. (2009). Multi-sensor data fusion with MATLAB. CRC press. 